\title{
Mucosal erosions in longterm non-steroidal anti-inflammatory drug users: predisposition to ulceration and relation to Helicobacter pylori
}

\author{
A S Taha, R D Sturrock, R I Russell
}

\begin{abstract}
The importance of erosions in longterm non-steroidal anti-inflammatory drug (NSAID) users, their relevance to ulceration and their relation to Helicobacter pylori are unclear. This study assessed the incidence of peptic ulcers in the presence or absence of erosions or $H$ pylori in a group of longterm NSAID users $(n=50)$, undergoing endoscopy at $0,4,12$, and 24 weeks while continuing with NSAIDs. Ulcers diagnosed at baseline endoscopy were excluded. Ulcers developed in nine of 23 patients (39\%) with pre-existing erosions compared with six of $27(22 \%)$ without erosions $(\mathbf{p}<0.05)$. The group infected with $H$ pylori $(n=30)$ had a total of 18 patients $(60 \%)$ with erosions, a total of 12 ulcers $(40 \%)$, and eight ulcers (27\%) complicating previous erosions, compared with five $(25 \%, p<0.01)$, three $(15 \%, p<0.05)$, and one $(5 \%, p<0.01)$ respectively in patients not infected with $H$ pylori $(n=20)$. Ulcer development was not influenced by the initial number of erosions but strongly associated with $H$ pylori positive duodenal erosions. It is concluded that ulcers are more likely to develop in longterm NSAID users who have mucosal erosions or $H$ pylori, or both.
\end{abstract}

(Gut 1995; 36: 334-336)

Keywords: mucosal erosion, non-steroidal antiinflammatory drug(s), Helicobacter pylori.

Gastric and duodenal erosions are the commonest endoscopic abnormalities related to acute exposure to non-steroidal anti-inflammatory drugs (NSAIDs). ${ }^{1}$ As they are commonly repaired through processes of restitution and adaptation, ${ }^{2}$ erosions are often transient, and this in turn leads to the impression that they are comparatively trivial lesions. ${ }^{3}$ Unlike the situation in subjects exposed to

Departments of Gastroenterology A S Taha R I Russell

and Rheumatology, Royal Infirmary, Glasgow

R D Sturrock

Correspondence to:

Dr A S Taha, GI Centre,

Southern General Hospital, Glasgow G51 4TF.

Glasgow G51 4TF. 24 June 1994 short courses of NSAIDs, the significance of

TABLE I Demographic details of the study groups

\begin{tabular}{lll}
\hline & Erosions present & Erosions absent \\
\hline Number & 23 & 27 \\
Men & 5 & 6 \\
Women & 18 & 21 \\
Age (y) median (interquartile & & \\
range) & $53(43-59)$ & $53(43-61)$ \\
Smokers & 10 & 11 \\
Alcohol drinkers & 13 & 16 \\
Upper abdominal complaints & 10 & 9 \\
\hline
\end{tabular}

TABLE II Characteristics of arthritis and details of anti-rheumatoid second line drugs

\begin{tabular}{lcc}
\hline & $\begin{array}{l}\text { Erosions present } \\
(n=23)\end{array}$ & $\begin{array}{l}\text { Erosions absent } \\
(n=27)\end{array}$ \\
\hline $\begin{array}{l}\text { Osteoarthritis } \\
\text { Rheumatoid arthritis }\end{array}$ & 3 & 5 \\
$\begin{array}{l}\text { Duration of arthritis (y) } \\
\text { median (interquartile range) }\end{array}$ & $5(3-11)$ & $7(3-11)$ \\
Second line drugs & & \\
$\quad$ Sulphasalazine & 8 & 10 \\
$\quad$ Gold (intramuscular) & 2 & 2 \\
$\quad$ Penicillamine & 0 & 1 \\
$\quad$ Hydroxychloroquine & 3 & 2 \\
\hline
\end{tabular}

erosions in longterm users is not clear. Also, their link to ulceration has not been proved, and their relation to Helicobacter pylori has not been defined.

We, therefore, aimed at investigating the development of peptic ulcers in a group of patients with chronic arthritis treated with NSAIDs in the presence or absence of erosions, and to assess the relevance of $H$ pylori to both erosions and ulcers in these patients.

\section{Patients and methods}

Patients with osteoarthritis or rheumatoid arthritis, and aged 18 years or over, were recruited from the Rheumatology Outpatient Clinic. NSAIDs had to be taken for at least four weeks before baseline endoscopy and to be continued throughout the study. Corticosteroids, cytotoxic drugs, or antiulcer treatment were not permitted. Endoscopy was performed after an overnight fast and intravenous midazolam was used for sedation.

Patients with ulcers at baseline endoscopy were excluded and those without ulcers were re-endoscoped at 4,12 , and 24 weeks. The primary end point was ulcer development at any stage of the study or the completion

TABLE III Details of NSAID intake

\begin{tabular}{lll}
\hline & $\begin{array}{l}\text { Erosions present } \\
(n=23)\end{array}$ & $\begin{array}{l}\text { Erosions absent } \\
(n=27)\end{array}$ \\
\hline Type & & \\
Diclofenac & 6 & 3 \\
Naproxen & 5 & 6 \\
Indomethacin & 4 & 7 \\
Ketoprofen & 2 & 2 \\
Fenbufen & 2 & 0 \\
Nabumetone & 1 & 3 \\
Flurbiprofen & 0 & 2 \\
Others & 3 & 4 \\
Dosetł & $3(2-3)$ & $3(2-3)$ \\
Duration (years) $\ddagger$ & $2(1-4)$ & $2(1-5)$ \\
\hline
\end{tabular}

$\star=$ One patient taking one of the following: ibuprofen, azapropazone, acematacin, or tiaprofenic acid. $t=$ Dosage scale: 1 , minimal dose; 2 , submaximal; 3 : maximal dose permitted. $\ddagger=$ Median (interquartile range). 
TABIE IV Numbers of patients with erosions or ulcers, in the presence (or absence) of $\mathrm{H}$ pylori at each endoscopy

\begin{tabular}{|c|c|c|c|c|}
\hline & Baseline & 4 Weeks & 12 Weeks & 24 Weeks \\
\hline All erosions $\star$ & 12 & 11 & 7 & 6 \\
\hline All ulcers & 0 & 8 & 5 & 2 \\
\hline Ulcers plus crosions & 0 & 2 & 3 & 1 \\
\hline Erosions & & & & \\
\hline H pylori present (absent) & $8(4)$ & $10(1)$ & $6(1)$ & $5(1)$ \\
\hline $\begin{array}{l}\text { Ulcers } \\
\text { H pylori present (absent) }\end{array}$ & 0 & $6(2)$ & $4(1)$ & $2(0)$ \\
\hline
\end{tabular}

$\star=$ Erosions developed in a total of 23 patients throughout the whole study, some of whom had lesions at more than one endoscopy (see text).

of the required series of endoscopic examinations. An ulcer was defined as a mucosal defect that is equal to or greater than $3 \mathrm{~mm}$ in diameter, ${ }^{4}$ and smaller lesions were considered as erosions. Ulcers were described as deep (more than $3 \mathrm{~mm}$ ) or comparatively superficial (2-3 $\mathrm{mm}$ in depth). Ulcer dimensions were measured using the standard Olympus upper gastrointestinal endoscopy biopsy forceps, with the fully open instrument being equivalent to $5 \mathrm{~mm}$. The anatomical locations of lesions were described as precisely as possible, their photographic appearances recorded, and their distance from the teeth measured. $H$ pylori was identified in gastric antral biopsy specimens by both culture and histological examination. The endoscopist was not aware of $H$ pylori status or the previous endoscopic findings, and the study patients were re-endoscoped among a larger pool of arthritic patients to facilitate blinding and further minimise familiarity between the endoscopist and patients being repeatedly endoscoped. Statistical analyses included the $\chi^{2}$ and Fisher's exact test where appropriate. The study was approved by the local ethics committee.

\section{Results}

A total of 50 patients were recruited. They were divided into two groups according to whether they had or had not had erosions at any stage of the study. Table I shows their demographic details. Patients in both groups were comparable with respect to their ages, sex, smoking, and drinking habits. Also, there were no significant differences in the characteristics of patients' arthritis, use of second line

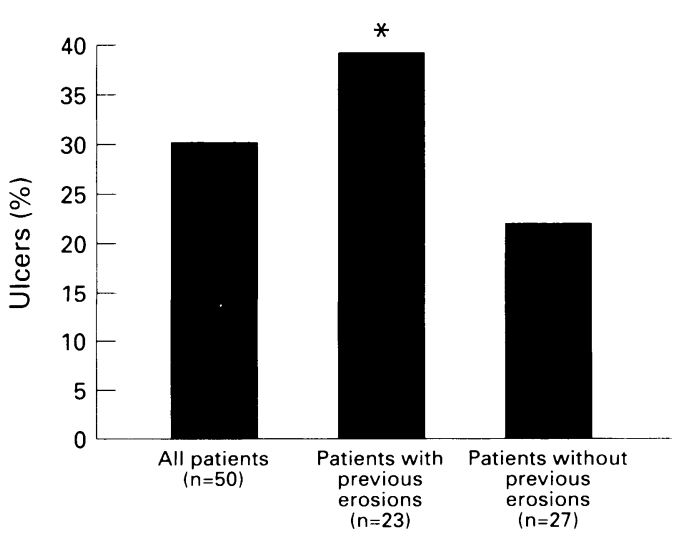

Figure 1: Ulcer development (\%) in patients with or without erosions. The presence of erosions was associated with a significant rise in subsequent ulceration. ${ }^{\star} p<0 \cdot 05$ (versus patients without erosions). drugs (Table II), or in the types, doses, or length of NSAID intake (Table III).

Table IV shows the numbers of patients with erosions or ulcers at each endoscopic assessment. The study protocol excluded patients with ulcers at baseline endoscopy. Erosions were identified in 12 patients at the initial endoscopy, and developed in 11 others on subsequent examinations. They were recurrent in five patients, and disappeared altogether in eight patients. At each assessment, lesions were more likely to be found in patients infected with $H$ pylori.

Figure 1 shows ulcers developing in patients with or without previous erosions. Fifteen ulcers were diagnosed in the study group as a whole ( 15 of $50,30 \%$ ), with a median size of 4 $\mathrm{mm}$ (range 3-10 $\mathrm{mm}$ ). Only five ulcers were comparatively superficial and most $(n=10)$ were deep. Ulcers affected the gastric body $(n=1)$, duodenum $(n=5)$, gastric antrum $(n=8)$, and both the duodenum and antrum $(\mathrm{n}=1)$. Most ulcers (nine of $15,60 \%$ ) developed in patients with previous or current erosions. The median number of erosions (per patient) appearing before an ulcer was three (range 1-6) compared with four (1-12) erosions not followed by ulceration. Direct progression from an erosion to an ulcer was thought to have taken place in six of nine ulcer patients with previous erosions.

Figure 2 shows ulcers and erosions classified according to the presence or absence of $H$ pylori. Patients with $H$ pylori infection $(\mathrm{n}=30)$ had significantly more erosions, ulcers, and ulcers complicating previous erosions than patients not infected with $H$ pylori $(\mathrm{n}=20)$. It is worth noting that of six patients with duodenal erosions found in the study group as a whole five were positive for $H$ pylori; all these five patients $(100 \%)$ developed ulceration at subsequent endoscopic examinations (two gastric and three duodenal ulcers). This is compared with only three ulcers developing in 13 patients $(23 \%)$ with $H$ pylori positive gastric erosions $(p<0.001)$. Also gastric submucosal haemorrhages were found in a total of five patients: three were infected with $H$ pylori, and two of these three subsequently developed gastric erosions.

\section{Discussion}

This study shows that longterm users of NSAIDs with mucosal erosions are more likely to develop ulceration than patients without these lesions. Also, the majority of both erosions and ulcers in longterm NSAID users develop in patients infected with $H$ pylori. The initial number of erosions in the individual patient does not seem to influence the risk of ulceration. In the presence of $H$ pylori, however, duodenal erosions have a stronger association with subsequent ulceration than their gastric counterparts.

A variety of types of injury develop after the administration of NSAIDs, ranging from petechial haemorrhages to erosions, and less commonly ulceration. The progression from the phase of submucosal haemorrhages to that 


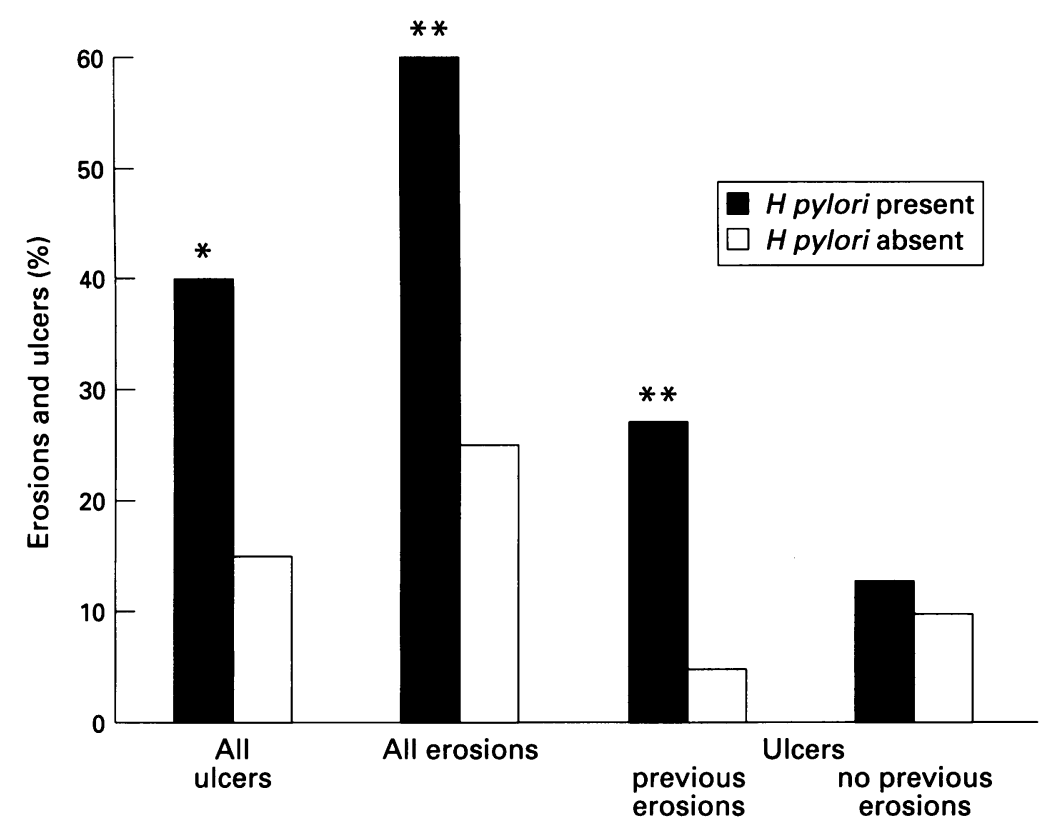

Figure 2: Erosions and ulcers (\%) in patients infected $(n=30)$ versus those not infected $(n=20)$ with $\mathrm{H}$ pylori. The overall proportions of ulcers and erosions, and ulcers complicating previous erosions were all higher in the presence of $\mathrm{H}$ pylori. ${ }^{\star} p<0.05$; $\star \star p<0 \cdot 01$. between our patients who developed ulcers and those who did not. This implies that the presence or absence of erosions is more useful than their numbers in predicting the risk of ulceration.

There have been very few prospective studies dealing with the possible interaction between NSAIDs and $H$ pylori. ${ }^{6}$ The findings of our study, which supports such interaction, are unlike those of another prospective study. ${ }^{7}$ This could be explained by the fact that both the duration of NSAID use and the follow up period of our patients were much longer than those of Lanza et al. ${ }^{7}$

It is also interesting to find that duodenal erosions in patients infected with $H$ pylori were more likely to predict ulceration than their gastric counterparts. The explanation for this is not clear but it might be related to differences between the gastric and the duodenal mucosa with respect to adaptation, blood flow, and the susceptibility to the damaging effects of $H$ pylori. ${ }^{8} 9$

In conclusion this study shows that peptic ulcers in longterm NSAID users are more likely to develop in patients with erosions despite the fact that such lesions might be transient or recurrent. Also, subsequent ulceration is more likely to be predicted by the presence of $H$ pylori, particularly in patients with duodenal erosions, than by the initial number of these lesions.

We wish to thank Professor F D Lee and Miss P Boothman for identification of $H$ pylori by histological examination and
culture respectively, and Mrs Ruth Simpson for her secretarial culture respe
assistance.

1 Lanza FL. A review of gastric ulcer and gastroduodenal injury in normal volunteers receiving aspirin and other injury in normal volunteers receiving aspirin and other non-steroidal anti-inflammatory drugs.

2 Graham DY, Smith JL, Spjut HJ, Torres E. Gastric adaptation: studies in humans during continuous aspirin administration. Gastroenterology 1988; 95: 327-33.

3 Laine L, Weinstein WM. Subepithelial haemorrhages and erosions of the human stomach. Dig Dis Sci 1988; 33: 490-503.

4 Graham DY, Agrawal NM, Roth SH. Prevention of NSAIDinduced gastric ulcer with misoprostol: multicentre double-blind, placebo-controlled trial. Lancet 1988; ii: 1277-80.

5 Bardhan KD, Bjarnason I, Scott DL, Griffin WM, Fenn GC Shield MJ, et al. The prevention and healing of NSAID damage by misoprostol. Br 7 Rheumatol 1993; 32: damage

6 Taha AS, Russell RI. Helicobacter pylori and non-steroidal anti-inflammatory drugs: uncomfortable partners in peptic ulcer disease. Gut 1993; 34: 580-3.

7 Lanza FL, Evans DG, Graham DY. Effect of Helicobacter pylori infection on the severity of gastroduodenal mucosa injury after the acute administration of naproxen and aspirin to normal volunteers. Am $\mathcal{F}$ Gastroenterol 1991; 86 $735-7$.

8 Taha AS, Angerson W, Nakshabendi I, Beekman H, Morran $\mathrm{C}$, Sturrock RD, et al. Gastric and duodenal mucosal blood $C$, Stur in pack flow in patients receiving non-steroidal anti-inflammatory drugs - influence of age, smoking, ulceration, and Helicobacter pylori. Aliment Pharmacol Therap 1993; 7 41-5

9 Taha AS, Dahill S, Nakshabendi I, Lee FD, Sturrock RD, Russell RI. Duodenal histology, ulceration, and Helicobacter pylori in the presence or absence of non-steroidal anti-inflammatory drugs. Gut 1993; 34: 1162-6. 